\title{
Linear baroclinic instability in the Martian atmosphere: Primitive equation calculations
}

\author{
H. L. Tanaka and Mayumi Arai \\ Institute of Geoscience, University of Tsukuba, Tsukuba 305-8571, Japan \\ (Received June 26, 1998; Revised November 16, 1998; Accepted December 18, 1998)
}

\begin{abstract}
In this study, baroclinic-barotropic instability of the Martian atmosphere is studied for a zonal basic state based on Mariner 9 observations, using a spherical linear primitive equation model derived from a method of 3-D normal mode expansion. As a result of solving a matrix eigenvalue problem, a distinctly unstable mode at synoptic to planetary scales was found with a peak growth rate of $2.3(1 / \mathrm{sol})$ at zonal wavenumbers $n=5$ to 6 with an eastward phase speed of $50(\% \mathrm{sol})$. The unstable mode has a period of $2.4 \mathrm{sol}$ for $n=3$, which agrees well with Viking observations. The geopotential amplitude maximum is located at $50^{\circ} \mathrm{N}$ near the surface, and the phase tilts westward with height. The structure is similar to baroclinic instability of a Charney mode associated with the subtropical jet on Earth. It is found, however, that the mode on Mars, where the subtropical jet is absent, is not the ordinary Charney mode, but a different one that is referred to as a monopole Charney mode in previous study which is characterized by its overall northward eddy momentum flux.
\end{abstract}

\section{Introduction}

According to observations by the Mariner 9 and Viking spacecraft, the Martian atmosphere appears to have active planetary-scale wavelike disturbances in the extratropics. Using Mariner 9 Infrared Interferometer Spectrometer (IRIS) data, Conrath (1981) detected temperature disturbance of wavenumber 2 in the Martian upper atmosphere during the late northern winter, assuming that the wave is stationary. This wave has an amplitude peak near $20 \mathrm{~km}$ altitude at $60^{\circ} \mathrm{N}$, and the phase tilts westward with respect to height. A possible source of such wavelike disturbances is stationary planetary waves forced by the large-scale surface topography. Hollingsworth and Barnes (1996) examined the cause of the wave- 2 disturbance using a spherical linear primitive equation model forced by the surface topography. They found that the northern extratropical eddies in their model exhibit a definite wavenumber 2 pattern, with comparable amplitude also at wavenumber 1 . The largest response in geopotential amplitudes is seen at $60^{\circ} \mathrm{N}$, but the vertical structure is essentially barotropic, with little vertical tilt.

As noted by Hollingsworth and Barnes (1996), however, other possible sources of the wavelike disturbances may be dynamical instabilities, such as baroclinic and/or barotropic instabilities. Barnes (1984) solved baroclinically mostunstable modes using a spherical quasi-geostrophic model by integrating in time. The fastest growing mode with a growth rate of $1.0 \mathrm{sol}^{-1}$ (sol is a Martian mean solar day of $88775 \mathrm{~s}$ ) was found at zonal wavenumbers 3 to 4 , with a phase speed of 10 to $20 \mathrm{~m} \mathrm{~s}^{-1}$. These results agree to some extent with in situ observations by the two Viking lan-

Copy right (C) The Society of Geomagnetism and Earth, Planetary and Space Sciences (SGEPSS); The Seismological Society of Japan; The Volcanological Society of Japan; The Geodetic Society of Japan; The Japanese Society for Planetary Sciences. ders although the phase speed is smaller than observations (Barnes, 1980, 1981). The structure of wavenumber 2 is characterized by an amplitude peak at $60^{\circ} \mathrm{N}$, with westward phase tilt with height. The maximum temperature amplitude was found to be located near the surface. Barnes (1984) suggested that the most unstable mode is essentially a Charneytype mode. These results should be confirmed using more sophisticated models. Michelangeli and Zurek (1987) investigated barotropic instability of midlatitude zonal jets. Although barotropic instability occurs for a narrow and strong jet, the period of the most unstable mode seems to be shorter than the Viking observation for the large Rossby number such as in Mars.

Previous studies have also employed general circulation models (GCM) to simulate the Martian atmosphere (e.g., Leovy and Mintz, 1969; Moriyama and Iwashima, 1980; Pollack et al., 1990; Barnes et al., 1993; Hollingsworth and Barnes, 1996; Wilson and Hamilton, 1996). Since Mars and Earth have similar astronomical parameters (e.g., rotation rate, size, atmospheric scale height), a well established technique developed for the terrestrial atmosphere may be applicable to the Martian atmosphere. Such investigations can provide a better understanding of extratropical disturbances in the Martian atmosphere. The simulated stationary waves in the extratropics exhibit a barotropic structure with pronounced southwest-northeast phase tilt at low levels during the northern winter (e.g., Barnes et al., 1993; Hollingsworth and Barnes, 1996). Travelling atmospheric waves are prominent, and remarkably regular, in northern midlatitudes during winter. Barnes et al. (1993) presented a result of GCM simulations with a focus on the transient baroclinic eddies. They analyzed a space-time power spectra of travelling waves in a Martian model atmosphere and found regularly propagating eddies of zonal wavenumbers 1 to 4 , exhibiting a constant eastward phase speed of approxi- 
mately $26 \mathrm{~m} / \mathrm{s}$ (about $60^{\circ} / \mathrm{sol}$ ) at $52.5^{\circ} \mathrm{N}$ in the northern winter. Wilson and Hamilton (1996) analyzed the thermotidal oscillations in the Martian atmosphere. In their model atmosphere, pronounced transient baroclinic waves were found in winter midlatitudes. Baroclinic waves of zonal wavenumbers $1-5$ propagate eastward with periods of 2-10 days, consistent with values deduced from the Viking lander data. In particular, zonal wavenumber 3 with a period of 2.2 days is especially prominent in midwinter. The origin of those transient eddies, however, is still unclear in terms of the sophisticated nonlinear model. More theoretical studies with linear model may be needed to understand the origin of these modes.

The linear baroclinic-barotropic instability has not been studied within the framework of the primitive equations. There has been limited study of dynamical instabilities with the primitive equations because spurious unstable gravity modes can grow rapidly in conjunction with geostrophic adjustment, obscuring meteorologically important modes. It is also well known that the time integration method used by Barnes (1984) has a difficulty in convergence if two different growing modes have similar growth rates. This is indeed the case for planetary waves in the terrestrial atmosphere. It is desirable to solve the problem using an eigenvalue approach with the primitive equations, using a realistic zonal basic state of the Martian atmosphere.

The purpose of this study is to examine baroclinicbarotropic instability of the Martian atmosphere using an eigenvalue approach with the spherical linear primitive equations. For that purpose, a method of 3-D normal mode expansion (see Tanaka and Kung, 1989) is applied in this study. The method is unique in that the high-frequency gravity modes can be removed effectively, retaining meteorologically relevant baroclinic-barotropic instability, especially for planetary-scale unstable modes. The basic state employed in this study is a zonal mean field for northern late winter compiled from Mariner 9 data by Hollingsworth and Barnes (1996), which is used for the study of forced stationary planetary waves. The growth rate, phase speed, and structure of unstable eigenmodes are presented and compared with the result obtained by Barnes (1984). The characteristics of baroclinic-barotropic instability for the Martian atmosphere are also compared with those found for the terrestrial atmosphere by Tanaka and Kung (1989). It is well known from previous studies that many features derived from linear theory are substantially altered by nonlinear dynamics: the most unstable mode is typically not the largest amplitude one, structures and phase speeds tend to change significantly in the nonlinear regime, etc. Nevertheless, a result from linear theory with less assumptions would help understand the process embedded behind and identify the origin of the transient eddies excited in the atmosphere.

\section{Model Description}

\subsection{Matrix notation of governing equations}

A system of primitive equations with spherical coordinates of longitude $\lambda$, latitude $\theta$, pressure $p$, and time $t$ may be reduced to three prognostic equations of horizontal motions and thermodynamics for three dependent variables of $U=(u, v, \phi)^{T}$. Here, $u$ and $v$ are the zonal and meridional components of the horizontal velocity $V$. The variable $\phi$ is a departure of the local isobaric geopotential from the reference state geopotential $\phi_{0}$, which is related through the hydrostatic equation to the reference state temperature $T_{0}$. Using a matrix notation, these governing equations (refer to Tanaka, 1991, 1998) may be written as

$$
M \frac{\partial U}{\partial t}+L U=N+F
$$

where

$$
\begin{aligned}
& U=(u, v, \phi)^{T}, \\
& M=\operatorname{diag}\left(1,1,-\frac{\partial}{\partial p} \frac{p^{2}}{R \gamma} \frac{\partial}{\partial p}\right), \\
& L=\left(\begin{array}{ccc}
0 & -2 \Omega \sin \theta & \frac{1}{a \cos \theta} \frac{\partial}{\partial \lambda} \\
2 \Omega \sin \theta & 0 & \frac{1}{a} \frac{\partial}{\partial \theta} \\
\frac{1}{a \cos \theta} \frac{\partial}{\partial \lambda} & \frac{1}{a \cos \theta} \frac{\partial() \cos \theta}{\partial \theta} & 0
\end{array}\right), \\
& N=\left(\begin{array}{c}
-V \cdot \nabla u-\omega \frac{\partial u}{\partial p}+\frac{\tan \theta}{a} u v \\
-V \cdot \nabla v-\omega \frac{\partial v}{\partial p}-\frac{\tan \theta}{a} u u \\
\frac{\partial}{\partial p}\left(\frac{p^{2}}{R \gamma} V \cdot \nabla \frac{\partial \phi}{\partial p}+\omega p \frac{\partial}{\partial p}\left(\frac{p}{R \gamma} \frac{\partial \phi}{\partial p}\right)\right)
\end{array}\right), \\
& F=\left(F_{u}, F_{v}, \frac{\partial}{\partial p}\left(\frac{p Q}{C_{p} \gamma}\right)\right)^{T} .
\end{aligned}
$$

The left-hand side of (1) represents linear terms with matrix operators $M$ and $L$ and the dependent variable vector $U$. The right-hand side represents a nonlinear term vector $N$ and a diabatic term vector $F$, which includes the zonal $F_{u}$ and meridional $F_{v}$ components of frictional forces and a diabatic heating rate $Q$. The parameter $a$ represents the radius of Mars, $\Omega$ the angular speed of the rotation, $R$ the specific gas constant, and $C_{p}$ the specific heat at a constant pressure. The static stability parameter $\gamma$ is defined as $\gamma=\frac{R T_{0}}{C_{p}}-p \frac{d T_{0}}{d p}$. The symbol $\nabla$ denotes the horizontal del-operator, $T$ the transpose of a matrix, diag the diagonal matrix, and $\omega=$ $d p / d t$ is diagnostically related to divergence.

In order to obtain a system of spectral equations, we expand the vectors $U$ and $F$ in 3-D normal mode functions in a resting atmosphere, $\Pi_{n l m}(\lambda, \theta, p)$ :

$$
\begin{gathered}
U(\lambda, \theta, p, t)=\sum_{n=-N}^{N} \sum_{l=0}^{L} \sum_{m=0}^{M} w_{n l m}(t) X_{m} \Pi_{n l m}(\lambda, \theta, p), \\
F(\lambda, \theta, p, t)=\sum_{n=-N}^{N} \sum_{l=0}^{L} \sum_{m=0}^{M} f_{n l m}(t) Y_{m} \Pi_{n l m}(\lambda, \theta, p) .
\end{gathered}
$$

Here, the expansion coefficients $w_{n l m}(t)$ and $f_{n l m}(t)$ are the functions of time alone. The subscripts represent zonal wavenumbers $n$, meridional indices $l$, and vertical indices $m$. They are truncated at $N, L$, and $M$, respectively. The expansion basis of the 3-D normal mode functions $\Pi_{n l m}$ is a tensor product of vertical structure functions $G_{m}$ and Hough harmonics $H_{n l m}$ (see Longuet-Higgins, 1968) which satisfies the following vertical structure equation and Laplace's tidal equation, respectively:

$$
-\frac{d}{d p}\left(\frac{p^{2}}{R \gamma} \frac{d G_{m}}{d p}\right)=\frac{1}{g h_{m}} G_{m},
$$




$$
\left(Y_{m}^{-1} L X_{m}\right) \Pi_{n l m}=i \sigma_{n l m} \Pi_{n l m},
$$

where the dimensionless eigenfrequency $\sigma_{n l m}$ is referred to as Laplace's tidal frequency, and $i$ is the imaginary unit. The scaling matrices should be defined for each vertical index as: $X_{m}=\operatorname{diag}\left(c_{m}, c_{m}, c_{m}^{2}\right)$, and $Y_{m}=\operatorname{diag}\left(2 \Omega c_{m}, 2 \Omega c_{m}, 2 \Omega\right)$, where $c_{m}=\sqrt{g h_{m}}$ is defined from the equivalent height $h_{m}$. The 3-D normal mode functions are known to form a complete set and satisfy an orthonormality condition under a proper inner product. Substituting (7) and (8) into (1), and using the orthonormality condition for the inner product, we obtain a system of 3-D spectral primitive equations in terms of the spectral expansion coefficients $w_{i}$ :

$$
\frac{d w_{i}}{d \tau}+i \sigma_{i} w_{i}=-i \sum_{j k} r_{i j k} w_{j} w_{k}+f_{i}, \quad i=1,2,3, \ldots,
$$

where $\tau$ is a dimensionless time scaled by $2 \Omega$, and $r_{i j k}$ is the interaction coefficient for nonlinear wave-wave interactions. The triple subscripts are shortened for simplicity such as $w_{n l m}=w_{i}$ and $f_{n l m}=f_{i}$. There should be no confusion in the use of $i$ for the subscript even though it is used also for the imaginary unit.

\subsection{Eigenvalue problem for linear instability}

The nonlinear spectral equation (11) is linearized for a prescribed zonal basic state in order to examine barotropicbaroclinic instability. A perturbation method is introduced using notations $\bar{w}_{i}$ for a time-independent zonal basic state and $w^{\prime}$ for small perturbations superimposed on the basic states (the same symbols with the original variables are used for convenience). The equation for the first-order term of perturbations for each zonal wavenumber becomes

$$
\begin{array}{r}
\frac{d w_{i}}{d \tau}+i \sigma_{i} w_{i}=-i \sum_{j=1}^{K}\left(\sum_{k=1}^{K}\left(r_{i j k}+r_{i k j}\right) \bar{w}_{k}\right) w_{j}, \\
i=1,2,3, \ldots, K,
\end{array}
$$

where the index $k$ is used for the basic state and $i$ and $j$ for the perturbations. Those indices are truncated at $K=$ $(L+1)(M+1)$ for each zonal wavenumber. It is straightforward to consider Newtonian cooling and Rayleigh friction with a common constant damping rate. Such a damping would simply reduce the growth rate of the unstable modes without altering the structure and phase speed. More complicated treatment would be necessary for spatially varying damping. As is commonly the case in the basic dynamical stability analysis, however, an inviscid and adiabatic case is considered in this study, disregarding the forcing and dissipation in perturbations. Likewise, we concentrate our analysis on the instability by the internal dynamics of the basic flow. Barnes et al. (1993) demonstrated that the impact of topography is important for the baroclinic eddies both in linear and nonlinear modeling. Such an impact of external forcing may be the future subject.

For a zonal basic state $\left(\bar{w}_{k} \neq 0\right.$ if $\left.n=0\right)$, we can rewrite the equation above in terms of a matrix form for each $n>0$ :

$$
\frac{d}{d \tau} W_{n}+i D_{n} W_{n}=-i B_{n} W_{n}, \quad n=1,2, \ldots, N,
$$

where $W_{n}=\left(w_{1}, \ldots w_{i}, \ldots w_{K}\right)^{T}$ and $D=\operatorname{diag}\left(\sigma_{1}, \ldots \sigma_{i}\right.$, $\left.\ldots \sigma_{K}\right)$. The $(i, j)$ entries of the real matrix $B_{n}$ is $\sum_{k=1}^{K}\left(r_{i j k}+\right.$ $\left.r_{i k j}\right) \bar{w}_{k}$ as appears in the right hand side of (12). Because (13) is linear, we can assume the solution of $W_{n}$ as:

$$
W_{n}(\tau)=\xi \exp (-i \nu \tau) .
$$

The initial value problem (13) is then reduced to an eigenvalue problem for a real matrix $D_{n}+B_{n}$ to obtain eigenvectors $\xi$ and eigenvalues $v$ as:

$$
\nu \xi=\left(D_{n}+B_{n}\right) \xi
$$

The eigenpairs are evaluated by a standard matrix eigenvalue solver. The eigenvalue $v$ provides information on the growth rate and phase speed of unstable modes, and the eigenvector $\xi$ represents the structure of the unstable modes by transforming it to the physical space with (7). In this model, the meridional indices involve both high-frequency gravity modes and low-frequency Rossby modes. For the terrestrial atmosphere, about $99 \%$ of atmospheric energy is contained in the Rossby modes, and the energy partitioned in gravity modes is at most $1 \%$ due to the geostrophic adjustment. The noisy high-frequency gravity modes are effectively truncated from the model and only the meteorologically relevant Rossby modes are retained to construct the model with the spectral truncations of $M=6$ and $L=20$.

\section{Results of Unstable Modes}

First, the vertical profile of the reference state temperature $T_{0}$ is obtained from observations by Viking 1 and 2 for a non-dusty case and is interpolated onto the 24 Gaussian levels as listed in Table 1 (refer to Seiff and Kirk, 1977). The mean surface pressure is set as $7.0 \mathrm{hPa}$, then the top pressure of the model atmosphere becomes $0.017 \mathrm{hPa}$ at $65 \mathrm{~km}$ height. The surface temperature is $223.3 \mathrm{~K}$ which decreases monotonically to $151.2 \mathrm{~K}$ at the model's top level. The static stability parameter $\gamma$ is approximately $25 \mathrm{~K}$ implying moderately stable stratification. Compared with the vertical temperature profile on Earth, a warmer layer associated with the stratosphere is missing on Mars due to the lack of an ozone layer.

The eigenvalue problem (9) is solved by the Galerkin Method (see Kasahara, 1984) with a 12 by 12 matrix using the vertical temperature profile in Table 1 . Twelve eigenmodes are obtained with different values of the equivalent depth $h_{m}$. The gravest mode $m=0$ indicates the equivalent depth of $12,989 \mathrm{~m}$ which agrees well with the result of non-dusty case by Zurek (1988) and is slightly larger than the value for Earth. The vertical structure functions for Mars (not shown) are quite similar to those for Earth, despite the lack of a stratospheric warmer layer. The Hough functions are then computed from (10) with the values of $h_{m}$. We find that there is no distinct difference in Hough functions between Earth and Mars. The diagonal matrix $D$ in (15) is obtained from the Laplace's tidal frequencies associated with the Hough functions for Mars.

Then, the matrix eigenvalue problem (15) is solved for a parameter set appropriate to the Martian atmosphere. The zonal mean basic state $\bar{w}_{k}$ examined in the study is compiled from the non-dusty case in Hollingsworth and Barnes (1996). Here, the zonal wind derived from observations by Mariner 9 is combined with results from GCM's in high altitudes for the northern late winter hemisphere. Figure 1 
Table 1. The vertical coordinate $\sigma$, pressure $p$, altitude $z$, temperature $T_{0}$, static stability parameter $\gamma$ at the 24 Gaussian levels.

\begin{tabular}{rrrrrr}
\hline & \multicolumn{1}{c}{$\sigma$} & $p(\mathrm{hPa})$ & $z(\mathrm{~km})$ & $T_{0}(\mathrm{~K})$ & $\gamma(\mathrm{K})$ \\
\hline 1 & -0.99 & 0.017 & 65.0 & 151.2 & 34.2 \\
2 & -0.97 & 0.088 & 47.1 & 158.5 & 26.7 \\
3 & -0.94 & 0.216 & 37.5 & 163.0 & 33.6 \\
4 & -0.89 & 0.398 & 30.9 & 171.7 & 15.8 \\
5 & -0.82 & 0.630 & 26.0 & 182.6 & 21.0 \\
6 & -0.74 & 0.910 & 22.0 & 188.0 & 32.7 \\
7 & -0.65 & 1.232 & 18.7 & 190.9 & 33.9 \\
8 & -0.55 & 1.591 & 16.0 & 193.5 & 34.6 \\
9 & -0.43 & 1.982 & 13.6 & 195.7 & 32.5 \\
10 & -0.32 & 2.397 & 11.6 & 198.7 & 27.0 \\
11 & -0.19 & 2.831 & 9.8 & 202.0 & 24.5 \\
12 & -0.06 & 3.276 & 8.2 & 205.4 & 25.0 \\
13 & 0.06 & 3.724 & 6.8 & 208.5 & 25.2 \\
14 & 0.19 & 4.169 & 5.6 & 211.1 & 25.7 \\
15 & 0.32 & 4.603 & 4.5 & 213.4 & 26.2 \\
16 & 0.43 & 5.018 & 3.6 & 215.5 & 26.7 \\
17 & 0.55 & 5.409 & 2.8 & 217.2 & 27.2 \\
18 & 0.65 & 5.768 & 2.1 & 218.7 & 27.1 \\
19 & 0.74 & 6.090 & 1.5 & 219.9 & 27.3 \\
20 & 0.82 & 6.370 & 1.0 & 221.0 & 27.1 \\
21 & 0.89 & 6.602 & 0.6 & 222.5 & 26.9 \\
22 & 0.94 & 6.784 & 0.3 & 222.9 & 26.9 \\
23 & 0.97 & 6.912 & 0.1 & 223.0 & 26.6 \\
24 & 0.99 & 6.983 & 0.0 & 223.2 & 26.5 \\
\hline
\end{tabular}

illustrates a latitude-height section of the zonal mean wind $\left(\mathrm{m} \mathrm{s}^{-1}\right)$ used in this study. The mean geopotential field is calculated from the zonal mean wind based on a gradient wind balance. We assume a symmetry about the equator for variables. The zonal flow has a maximum wind speed of $100 \mathrm{~m} \mathrm{~s}^{-1}$ at $60^{\circ} \mathrm{N}, 50 \mathrm{~km}$ height. There is no subtropical jet near $30^{\circ} \mathrm{N}$, unlike Earth. Although the structure involves significant uncertainty, especially at high altitudes, it may be our best estimate of non-dusty case based on the limited observations.

Given the zonal basic state, we can calculate the matrix $B_{n}$ and solve the eigenvalue problem of (15) for each zonal wavenumber. Figure 2 plots growth rates $(1 / \mathrm{sol})$ and phase speeds $(\% \mathrm{sol})$ of the unstable modes as functions of zonal wavenumbers. The fastest growing mode is designated as $M_{1}$, the second fastest growing modes as $M_{2}$, and so forth. The results show a distinctly unstable mode $M_{1}$ over the wavenumbers 1 through 11 indicating a peak at $n=5$ to 6 . The peak growth rate $2.3(1 / \mathrm{sol})$ is 5 times larger than the most unstable baroclinic instability calculated for Earth. It is twice as large as that obtained by Barnes (1984) for Mars, probably due to the lower static stability of the reference state used here. The fastest growing mode travels eastward with a phase speed of $50(\% / \mathrm{sol})$ for almost all waves. The mode thus rotates Mars once a week. The eastward phase speed of $M_{1}$ is twice as fast as those by Barnes (1984) and is 5 times faster than most unstable modes for Earth which rotate once a month. The phase speed coincides fairly well with the GCM simulation for the northern winter case by Barnes et al. (1993). The dominant waves are, however, not $n=5$ to 6 but $n=2$ to 4 in the nonlinear model. It is well known that the most unstable mode is not necessarily the largest amplitude mode. The linear model should have clear limitation in its application. The nonlinear model tends to show greater amplitudes in planetary waves than in synoptic waves because the fast growing synoptic waves tend to break down sooner than planetary waves. Another reason is that the wave breaking at synoptic waves causes the nonlinear wavewave interaction of energy from synoptic to planetary waves. Those processes are beyond the scope of the linear theory.

The periods for $M_{1}$ are 6.0, 3.4, 2.4, 1.7, and $1.4 \mathrm{sol}$ for $n=$ 1 to 5 , respectively. According to the observation by Viking Lander $2\left(\sim 48^{\circ} \mathrm{N}\right)$, frequency spectra of surface pressure indicate peaks at $6.7,3.4$, and $2.4,1.7$, and 1.4 sol for a late autumn-winter period (see Fig. 3 after Barnes, 1981). The agreement is remarkably well for $n=2$ to 5 . The observed largest spectral peak at the period of 2.4 sol in Fig. 3 is very likely to be the $M_{1}$ mode at wavenumber $n=3$. Likewise, the observed peaks at $6.7,3.4,1.7$, and 1.4 sol correspond well with $M_{1}$ mode at wavenumbers $n=1,2,4$, and 5, respectively. The observed spectral peak at $6.7 \mathrm{sol}$ may correspond to the period for $n=1$, but the modal features predicted by linear theory must be altered substantially by the nonlinear wave-wave interactions.

The second unstable mode $M_{2}$ has a growth rate of 1.2 ( $1 / \mathrm{sol})$ at $n=4$ to 5 . This mode is detected over the zonal waves $n=1$ to 8 . The phase speed is approximately constant, at $45(\% \mathrm{sol})$, but does tend to decrease as $n$ increases. There are also much slower growing modes $M_{3}, M_{4}$, and so forth, but these modes should be less important because of their small growth rates. Introducing frictional damping would reduce the growth rate and alter the structure and phase speed of the eigen modes. We can infer the effect of such damping by introducing Rayleigh friction and the comparable amount of Newtonian cooling, which alters (reduces) the growth rate by the given amount of damping without altering the phase speed and the structure of the eigen modes. Therefore, slowly growing modes are no longer unstable under the frictional damping.

Figure 4 illustrates the latitude-height section of geopotential for the most unstable mode $M_{1}$ of zonal wavenumbers $n=1,2,3$ and for $n=4,6,8$. The amplitude is plotted by solid lines and the phase by dashed lines. The phase is expressed by the east longitude of the first ridge in degree. The amplitude peak is located at the top of the model domain in high latitudes for $n=1$. Another separated peak is seen near the surface at $65^{\circ} \mathrm{N}$. The phase tilts westward with height. For $n=2$ the upper-air amplitude peak near $20 \mathrm{~km}$ is comparable to the surface peak. Hartmann (1979) presented a similar meridional structure of the unstable mode of planetary waves, using quasi-geostrophic mode for the terrestrial model. He demonstrated that the low-wavenumber mode is deep, indicating its amplitude maximum in high altitudes and is still considered as Charney type modes. For $n=3$ the upper-air peak disappears and the maximum is now confined near the surface. It is shown that only planetary waves 


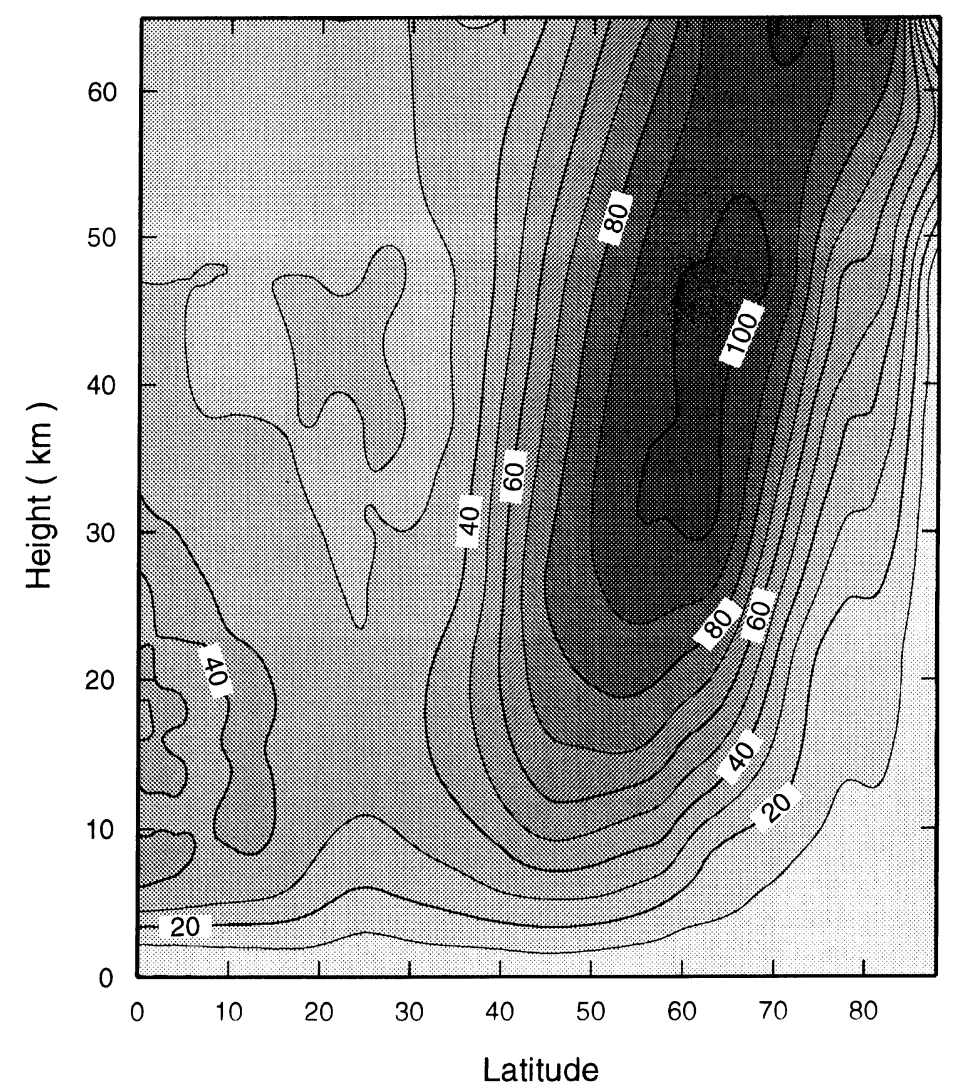

Fig. 1. The latitude-height section of the zonal mean wind $\left(\mathrm{m} \mathrm{s}^{-1}\right)$ in the northern winter hemisphere, reproduced from Hollingsworth and Barnes (1996). The zonal flow has a maximum wind speed of $100 \mathrm{~m} \mathrm{~s}^{-1}$ at $60^{\circ} \mathrm{N}, 50 \mathrm{~km}$ height.

can propagate in the vertical showing large amplitudes in the upper-air. In contrast, shorter waves are confined near the surface. The structure is quite similar to that found by Barnes (1984).

The phases for $n=1,2,3$ tilt westward with height, especially in the northern side of the amplitude peak. The westward phase tilt implies a northward eddy heat flux and thus the mode is excited by baroclinic instability. The eddy heat flux is downgradient so that the zonal available potential energy is converted to eddy available potential energy and to kinetic energy in midlatitudes. On the other hand, the phase tilts eastward with respect to latitudes, implying northward eddy momentum flux over almost the entire domain. The eddy momentum flux is downgradient against the mean flow in high latitudes, although it is upgradient in mid to low latitudes. Thus, we might expect contributions from barotropic instability to some extent in addition to the baroclinic energy supply for the fastest growing mode $M_{1}$.

The structures for $n=4,6,8$ are qualitatively similar to that of $n=3$. The amplitude peak is confined near the surface at $50^{\circ} \mathrm{N}$, and the upper-air peak does not exist. The westward vertical phase tilt is a common feature for each zonal wave, whereas the meridional phase tilt tends to diminish for $n=4$ to 8 . It is interesting to note that the geopotential amplitude does not penetrate equatorward of $30^{\circ} \mathrm{N}$ for all modes at low level. The pressure variation observed by Viking Lander $2\left(\sim 48^{\circ} \mathrm{N}\right)$ is $2 \sim 5$ times larger than that of Lander $1\left(\sim 23^{\circ} \mathrm{N}\right)$ as documented by Barnes (1980). In this regard, the structure in Fig. 4 is consistent with the observations. Similar result was obtained by the former linear stability analysis (see Barnes, 1984). In contradiction, nonlinear GCM studies indicate overly large subtropical amplitude of disturbances in lowland regions (see Barnes et al., 1993). The reason of the contradiction in the nonlinear model is still unclear.

It should be noted that the overall northward eddy momentum flux for the mode $M_{1}$ is distinct from baroclinic instability $M_{\mathrm{c}}$ on Earth (the ordinary Charney mode) which tends to converge eddy westerly momentum flux into midlatitudes supported by the equatorward momentum flux in high latitudes. In the terrestrial atmosphere, the Charney mode $M_{\mathrm{c}}$ is mainly excited by the westerly shear associated with the subtropical jet near $30^{\circ} \mathrm{N}$. According to Tanaka and Kung (1989), the $M_{1}$ is referred to as a monopole Charney mode and is distinctly separated from $M_{\mathrm{c}}$ modes by an eigenvalue analysis of the terrestrial atmosphere. The $M_{2}$ mode is identified as a dipole Charney mode as will be discussed later. Here, monopole and dipole are distinguished by the amplitude peaks in geopotential distributions. There is a socalled Green mode $M_{\mathrm{G}}$ on Earth which is identified as the first internal mode with one nodal structure in the vertical (Green, 1960). On this basis, $M_{1}, M_{2}$, and $M_{\mathrm{c}}$ are all recognized as a family of Charney modes which has a common property of an external mode without any apparent node in 

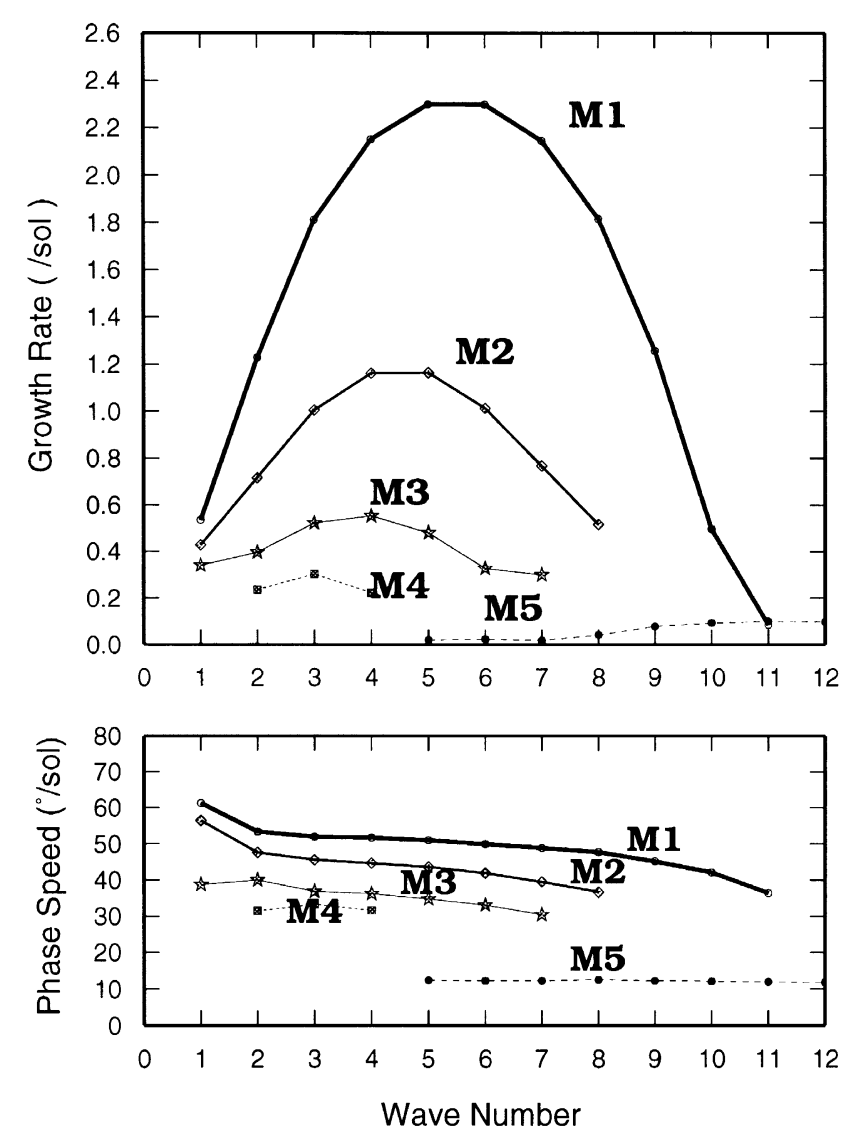

Fig. 2. Growth rates (1/sol) and phase speeds ( $\%$ sol) of the unstable modes as functions of zonal wavenumber. The fastest growing mode is designated as $M_{1}$, the second fastest growing modes as $M_{2}$, and so forth.

the vertical. Contrasted with the beta-plane geometry, the spherical geometry allows the Charney modes to choose different meridional structure. $M_{1}$ and $M_{\mathrm{c}}$ modes have a single amplitude peak in the midlatitude, whereas $M_{2}$ has two amplitude peaks with a characteristic nodal structure in the meridional. The difference between $M_{1}$ and $M_{\mathrm{c}}$ modes is realized in the different phase structures. $M_{\mathrm{c}}$ mode shows converging momentum flux in midlatitudes with northward flux in the southern flank and southward flux in the northern flank of the amplitude peak. $M_{1}$ mode, on the other hand, indicates overall northward momentum flux. Although both $M_{1}$ and $M_{\mathrm{c}}$ have a single amplitude peak in the meridional, the meridional scale of $M_{1}$ is twice as large as $M_{\mathrm{c}}$ in terms of the phase structure.

Although previous studies identify the most unstable mode on Mars as the ordinary Charney mode $M_{\mathrm{c}}$ (e.g., Barnes, 1984), mode like that for Earth $M_{\mathrm{c}}$ is unlikely to occur in the Martian atmosphere because a subtropical jet is absent. It is therefore reasonable to recognize the most unstable mode on Mars as the monopole Charney mode $M_{1}$ from its structure and the energy source. Barnes (1984) reported in his study that the momentum fluxes of the unstable Charney modes on Mars are almost entirely poleward, which differs from the terrestrial typical Charney mode. In this respect, the discrepancies in the structure is solved in this study.

Figure 5 illustrates a geopotential field as in Fig. 4, but for
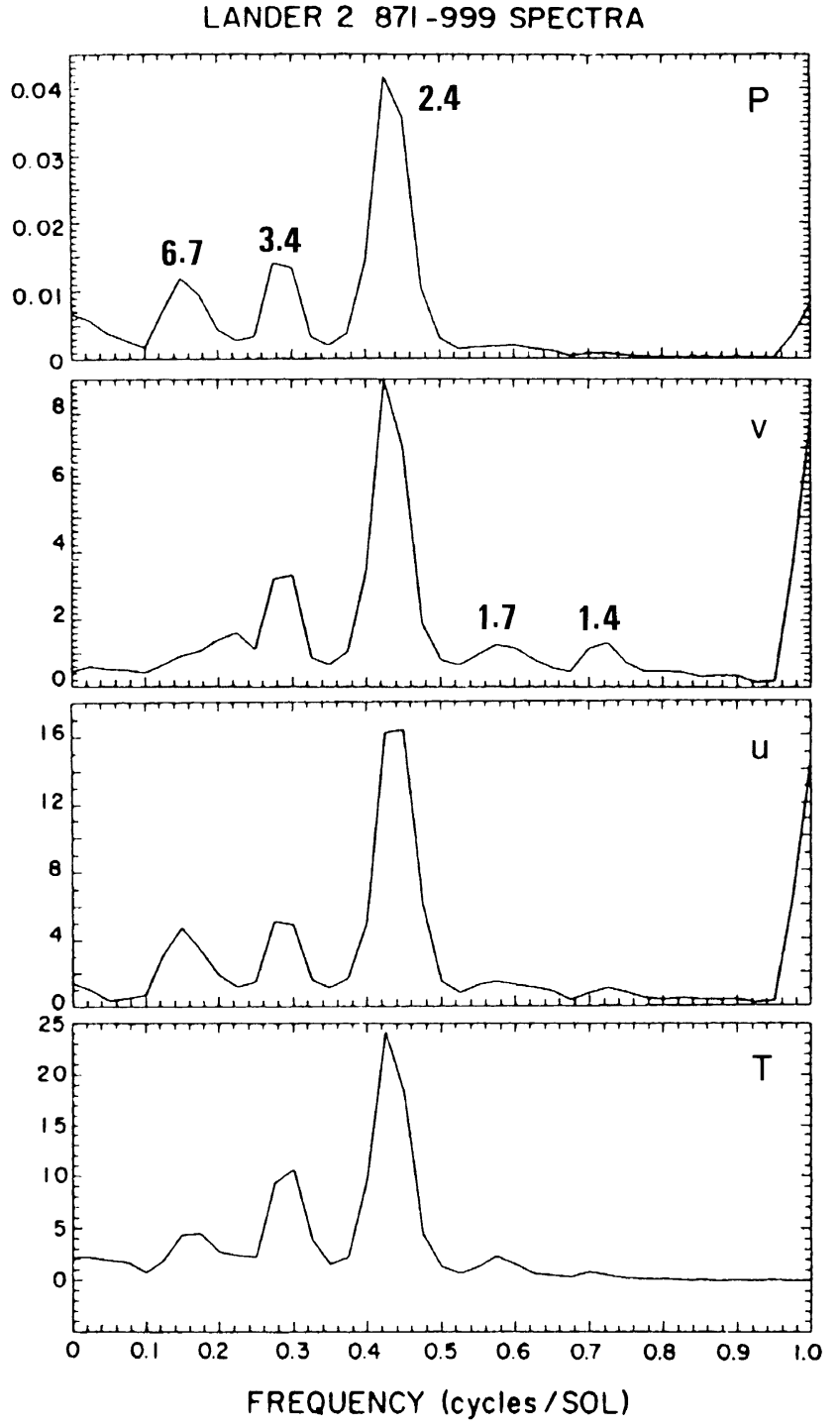

Fig. 3. The pressure, wind and temperature spectra of in situ observations by Viking Lander 2 at $\sim 48^{\circ} \mathrm{N}$ (after Barnes, 1981). The numbers along the spectral peaks indicate the period ( $\mathrm{sol})$.

the second most unstable mode $M_{2}$ for zonal wavenumbers $n=2,4,6$. The notable characteristics of this mode are seen in the meridional dipole structure. The amplitude peaks split in high and low latitudes at $45^{\circ} \mathrm{N}$ and $60^{\circ} \mathrm{N}$ near the surface. The ridge locations of these two peaks are out of phase with each other, as seen from sharp transition of the phase between the two peaks. The southern amplitude peak has a westward phase tilt with height, whereas the tilt is almost absent for the northern amplitude peak. This mode is excited by the northward eddy heat flux in the southern part of the dipole structure, and is identified as dipole Charney mode $M_{2}$ by Tanaka and Kung (1989) which is related to dipole blocking on Earth. In any case, this mode is probably less important than $M_{1}$ mode due to the smaller growth rate.

\section{Concluding Remarks}

In this study, baroclinic-barotropic instability of the Martian atmosphere is analyzed, using a spherical linear primitive equation model derived from a method of 3-D normal mode 


\section{$\operatorname{M1}(\mathbf{n}=1)$}

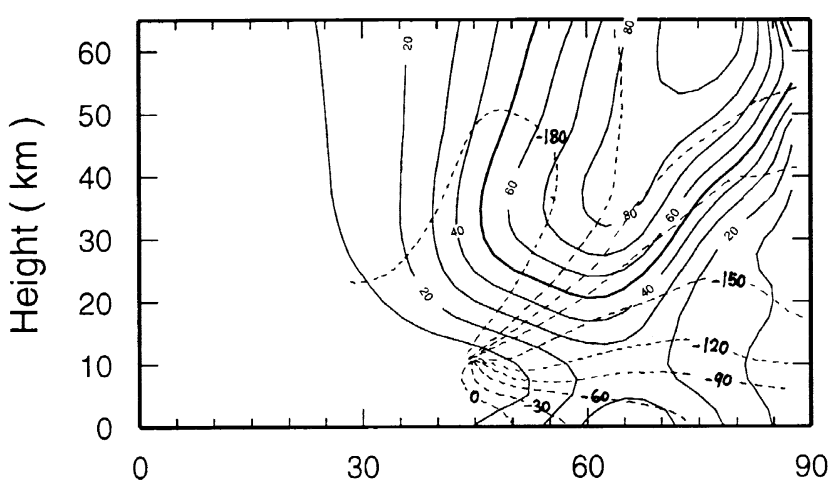

M1 ( $\mathbf{n}=\mathbf{2}$ )

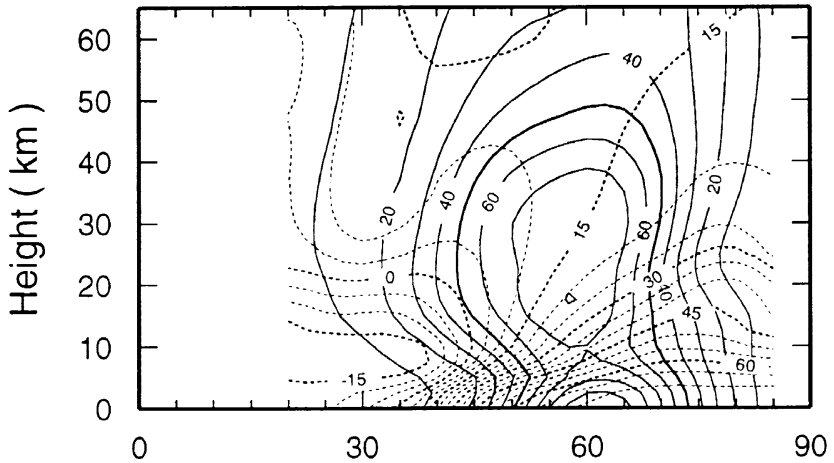

$\mathbf{M 1}(\mathbf{n}=\mathbf{3})$

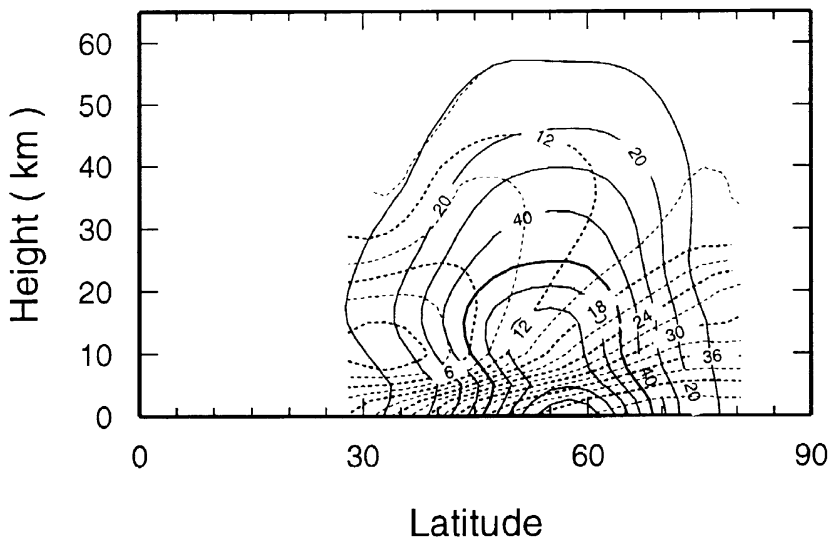

Fig. 4. The latitude-height section of geopotential amplitude (solid line) and phase (dashed line, indicating the east longitude of the first ridge in degree) of the fastest growing mode $M_{1}$ for zonal wavenumbers $n=1$, 2,3 , and for $n=4,6,8$.

expansion. The basic state analyzed in this study is a zonal mean field of northern late winter compiled from Mariner 9 by Hollingsworth and Barnes (1996).

As a result of the eigenvalue problem, we found a most unstable mode $M_{1}$ at zonal wavenumbers $n=1$ to 11 with a peak growth rate of $2.3(1 / \mathrm{sol})$ at $n=5$ to 6 and an eastward phase speed $50(\%$ sol). The growth rate is twice as large as that obtained by Barnes (1984). The structure of the $M_{1}$ mode for $n=6$ exhibits a peak amplitude at $50^{\circ} \mathrm{N}$ near the surface with a westward phase tilt in the vertical. That for $n=2$ has a geopotential amplitude peak at $60^{\circ} \mathrm{N}$ near $20 \mathrm{~km}$
M1 ( $n=4)$

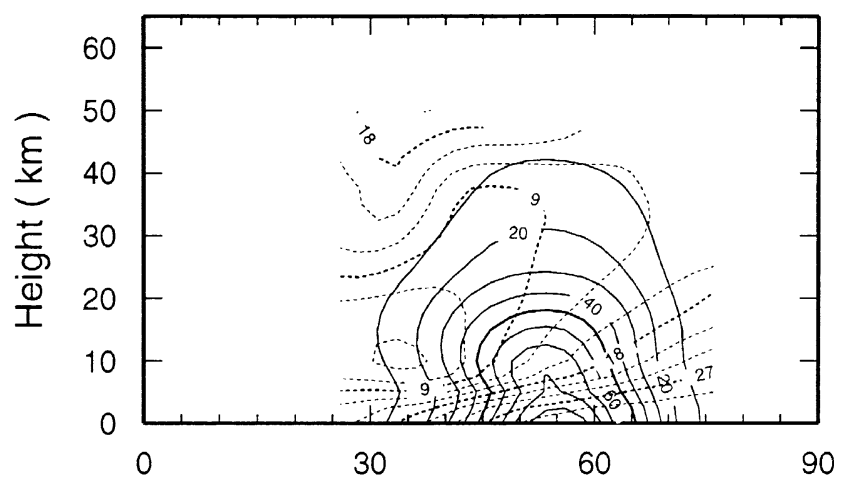

$M 1(n=6)$
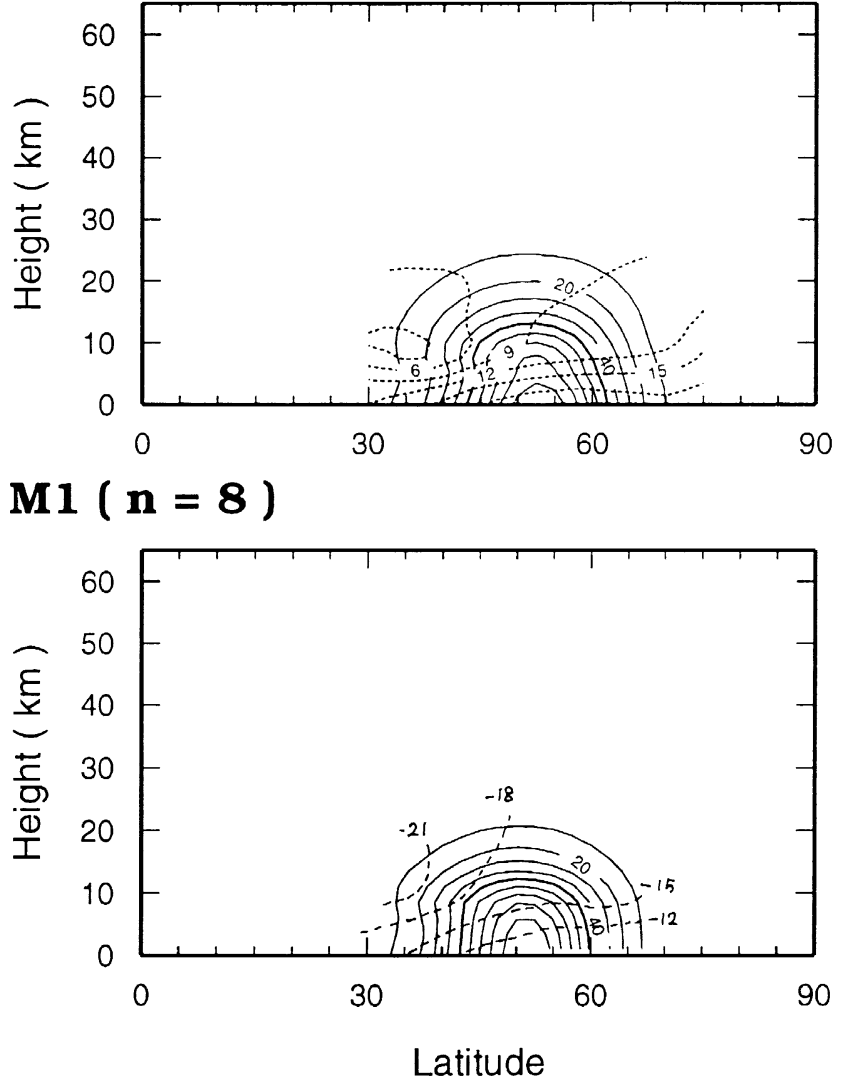

Fig. 4. (continued).

altitude with a westward vertical phase tilt. The structure is consistent with Barnes' results, and the wave is evidently excited by baroclinic instability. The periods of the $M_{1}$ mode are $6.0,3.4,2.4,1.7$, and 1.4 sol for $n=1$ to 5 , respectively. Those periods agree remarkably well with Viking Lander 2 in situ observation. The largest amplitude is seen, however, at $n=3$ in observation and in GCM results by Wilson and Hamilton (1996) and Barnes et al. (1993), probably due to the nonlinear effect.

We can identify this $M_{1}$ mode on Mars with a monopole Charney mode $M_{1}$ in the terrestrial atmosphere, as detected by Tanaka and Kung (1989) for $n=1$ to 5 . The meridional structure of $M_{1}$ is distinctly different from that of ordinary Charney mode $M_{\mathrm{c}}$ in that the eddy momentum flux is essen- 
$\mathbf{M 2}(\mathbf{n}=\mathbf{2})$

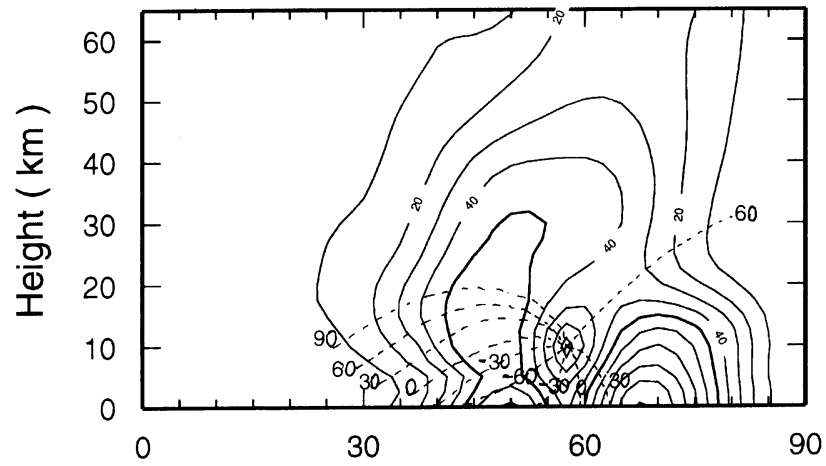

M2 ( $n=4)$

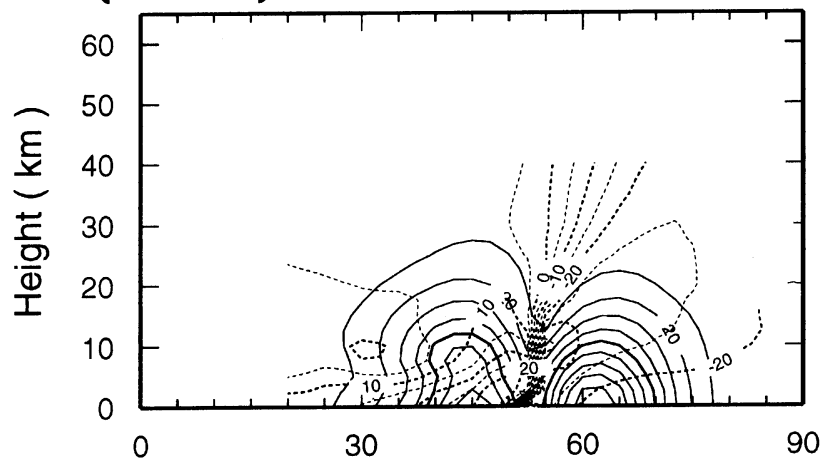

$\operatorname{M2}(n=6)$

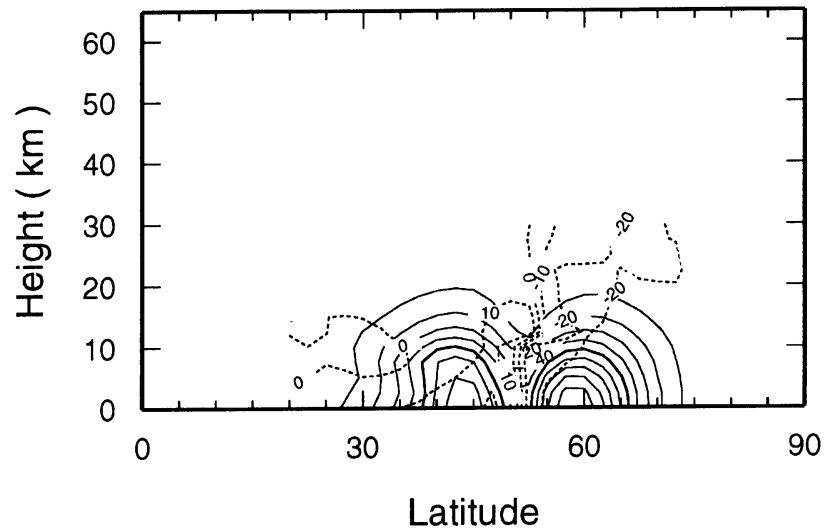

Fig. 5. As in Fig. 4, but for the second fastest growing mode $M_{2}$.

tially northward. The $M_{\mathrm{c}}$ mode on Earth, in contrast, tends to converge eddy westerly momentum flux into midlatitudes supported by equatorward momentum flux in high latitudes. The monopole Charney mode $M_{1}$ obtained in this study is induced by the upper-air westerlies associated with the polar vortex near $60^{\circ} \mathrm{N}$. Therefore, it is different from the ordinary Charney mode $M_{\mathrm{c}}$ which is induced mainly by the subtrop- ical jet near $30^{\circ} \mathrm{N}$ on Earth. Further study may be desirable to compare the present results with the dusty case of stronger basic flow. The influence of the different static stability for the dusty case upon the instability should be assessed in the future study.

Acknowledgments. This research was supported by the Grant-inAid for Scientific Research, Japanese Ministry of Education, Science, Sports and Culture, No. 09640518. The authors appreciate Mr. M. Miura, Mr. D. Nohara and Ms. K. Honda for their technical assistance.

\section{References}

Barnes, J. R., Time spectral analysis of midlatitude disturbances in the Martian atmosphere, J. Atmos. Sci., 37, 2002-2015, 1980.

Barnes, J. R., Midlatitude disturbances in the Martian atmosphere: A second Mars year, J. Atmos. Sci., 38, 225-234, 1981

Barnes, J. R., Linear baroclinic instability in the Martian atmosphere, $J$. Atmos. Sci., 41, 1536-1550, 1984.

Barnes, J. R., J. B. Pollack, R. M. Haberle, C. B. Leovy, R. W. Zurek, H. Lee, and J. Schaeffer, Mars atmospheric dynamics as simulated by the NASA Ames general circulation model, 2. Transient baroclinic eddies, J. Geophys. Res., 98, 3125-3148, 1993.

Conrath, B. J., Planetary-scale wave structure in the Martian atmosphere, Icarus, 48, 246-255, 1981.

Green, J. S. A., A problem in baroclinic instability, Quart. J. Roy. Meteor Soc., 86, 237-251, 1960.

Hartmann, D. L., Baroclinic instability of realistic zonal-mean states to planetary waves, J. Atmos. Sci., 36, 2336-2349, 1979.

Hollingsworth, J. L. and J. R. Barnes, Forced stationary planetary waves in Mars's winter atmosphere, J. Atmos. Sci., 53, 428-448, 1996.

Kasahara, A., The linear response of a stratified global atmosphere to tropical thermal forcing, J. Atmos. Sci., 41, 2217-2237, 1984.

Leovy, C. B. and Y. Mintz, Numerical simulation of the atmospheric circulation and climate of Mars, J. Atmos. Sci., 26, 1167-1190, 1969.

Longuet-Higgins, M. S., The eigenfunction of Laplace's tidal Equation over a sphere, Phil. Trans. Roy. Soc., London, A262, 511-607, 1968.

Michelangeli, D. V. and R. W. Zurek, Barotropic instability of midlatitude zonal jets on Mars, Earth and Venus, J. Atmos. Sci., 44, 2031-2041, 1987.

Moriyama, S. and T. Iwashima, A spectral model of the atmospheric general circulation of Mars: A numerical experiment including the effects of the suspended dust and the topography, J. Geophys. Res., 85, 2847-2860, 1980.

Pollack, J. B., R. M. Baberle, J. Schaeffer, and H. Lee, Simulation of the general circulation of the Martian atmosphere, I: Polar processes, J. Geophys. Res., 95, 1447-1474, 1990.

Seiff, A. and D. B. Kirk, Structure of the atmosphere on Mars in summer at mid-latitudes, J. Geophys. Res., 82, 4364-4378, 1977.

Tanaka, H. L., A numerical simulation of amplification of low-frequency planetary waves and blocking formations by the upscale energy cascade, Mon. Wea. Rev., 119, 2919-2935, 1991.

Tanaka, H. L., Numerical simulation of a life-cycle of atmospheric blocking and the analysis of potential vortisity using a simple barotropic model, $J$. Meteor. Soc. Japan, 76, 983-1008, 1998.

Tanaka, H. L. and E. C. Kung, A study of low-frequency unstable planetary waves in realistic zonal and zonally varying basic states, Tellus, $\mathbf{4 1 A}$ 179-199, 1989.

Wilson, R. J. and K. Hamilton, Comprehensive model simulation of thermal tides in the Martian atmosphere, J. Atmos. Sci., 53, 1290-1326, 1996.

Zurek, R. W., Free and forced modes in the Martian atmosphere, J. Geophys. Res., 93, 9452-9462, 1988.

H. L. Tanaka (e-mail: tanaka@atm.geo.tsukuba.ac.jp) and M. Arai 